ISSN 1870-4069

\title{
Finding Events in Background EEG in Rats in Early Posttraumatic Period
}

\author{
Ivan A. Kershner ${ }^{1}$, Yurii V. Obukhov ${ }^{1}$, Ilya G. Komoltsev ${ }^{2}$ \\ ${ }^{1}$ Kotel'nikov Institute of Radio Engineering and Electronics of RAS, \\ Moscow, Russia \\ ${ }^{2}$ Institute of Higher Nervous Activity and Neurophysiology of RAS, \\ Moscow, Russia
}

\begin{abstract}
Processing of a big experimental data is a problem in neurophysiologic researches. Epileptiform discharges (ED) detection in long EEG-records requires an algorithm for recognition of ED and sleep spindles (SS). Electroencephalogram recordings were performed in rats using implanted electrodes before and after traumatic brain injury (TBI). Intervals about 10 second with typical ED and typical SS have been manually selected from these records by experts. The algorithm for the allocation of events from background activity was developed. Parameters founded by the algorithm can serve as criteria for the recognition of ED and SS events.
\end{abstract}

Keywords: EEG, rats, event detection.

\section{Introduction}

Posttraumatic epilepsy (PE) is a serious medical and social problem. It develops in $2-17 \%$ of patients after traumatic brain injury (TBI) [1]. It takes years for a posttraumatic epileptogenesis to develop and its mechanisms are poorly understood. At the same time, prediction of PE in clinical practice is the unsolved problem [9]. Animal models [11,6] are widely used to study neurobiological mechanisms of epileptogenesis to detect biomarkers for PE development and to find new targets for drugs to prevent $\mathrm{PE}$.

$\mathrm{PE}$ induced by lateral fluid percussion (LFP). Head trauma reproduces clinical signs and, most likely, pathogenesis of PE [10]. Silent period of epileptogenesis from TBI to first unprovoked seizure in rats takes weeks. Many groups using this model are focused on delayed consequences appearing weeks and months after TBI [3]. However, appearance of early epileptiform discharges (ED) on electroencephalograms (EEG) and its dynamics are possible biomarkers of PE development [4].

So, it has been suggested that the appearance and dynamics of development of ED in early posttraumatic period can predict the development of PE. To verify this hypothesis a detailed analysis of long EEG-records is needed. Total duration of EEG-records from one experimental group, which consists of 12 experimental 
and 12 sham-operated animals with 2 weeks EEG length per animal, is one year and it is not the limit. Therefore, automatic detection of ED is essential for processing big data and we focused on this problem.

TBI was modeled on Wistar rats using lateral fluid percussion of $3 \mathrm{~atm}$. [7]. To detect ED four stainless steel epidural electrodes were implanted. One week prior to and one week after TBI the video-electrocorticograms were recorded.

\section{Method of Detection}

Long EEG-records include all phases of wake-sleep cycle of the animals. Sleep spindles (SS) are a normal physiological EEG-pattern of sleep. One sign of ED is the increase of amplitude, which is also present in SS that make difficult to automatically recognize ED. Figure 1 shows example of ED and of SS, respectively, on the day following TBI.
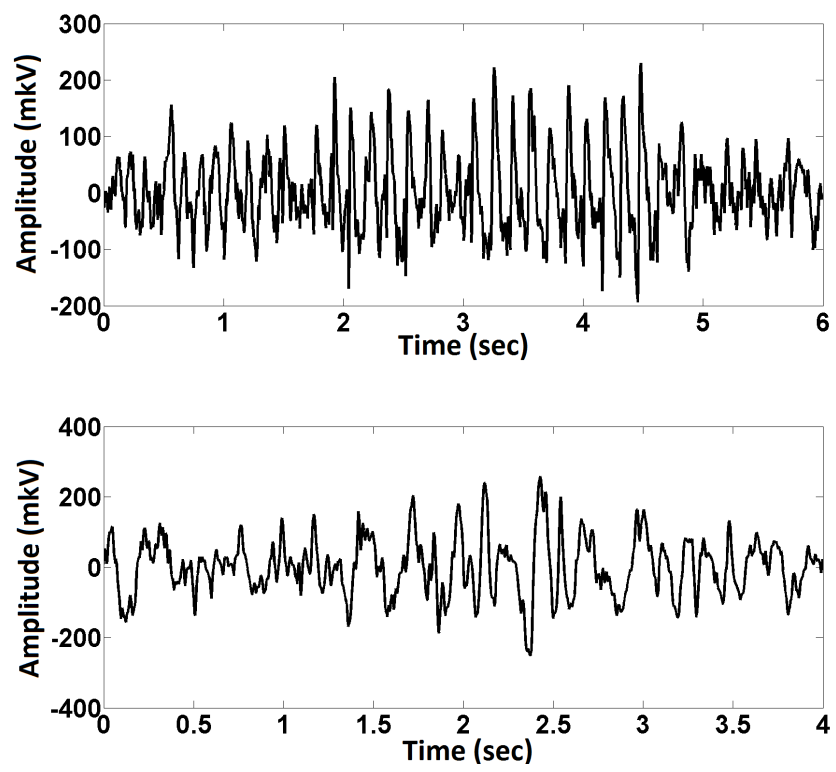

Fig. 1. Upper plot: epileptic discharge; lower plot: sleep spindles.

Algorithm of finding ED has to recognize SS and separate it from ED to avoid wrong positives results. To solve this problem, fragments of the EEG lasting from 3 to 10 seconds was allocated from daily record by neurophysiologists. They contain ED or SS. 36 SS and 39 ED were selected to be compared.

All fragments were processed by the 8th order bandpass Butterworth filter 2-124 Hz and a set of notch filters were applied for removing a power line noise 
at 50 and $100 \mathrm{~Hz}$ from signals recorded with a sampling rate $250 \mathrm{~Hz}$. After that, the complex Morlet wavelet was used [5]:

$$
\begin{gathered}
S_{x}=|W(\tau, f)|^{2}, \\
W(\tau, f)=\frac{1}{f} \int x(t) \psi *\left(\frac{t-\tau}{f}\right), \\
\psi(\eta)=\frac{1}{\sqrt{\pi F_{b}}} e^{2 \pi \imath F_{c} \eta} e^{\frac{-\eta^{2}}{F_{b}}}
\end{gathered}
$$

The equation (1) describes the power spectral density (PSD). The wavelet transform (2) is based on the Morlet wavelet (3). The coefficients $F_{b}$ and $F_{c}$ equal to 1 .
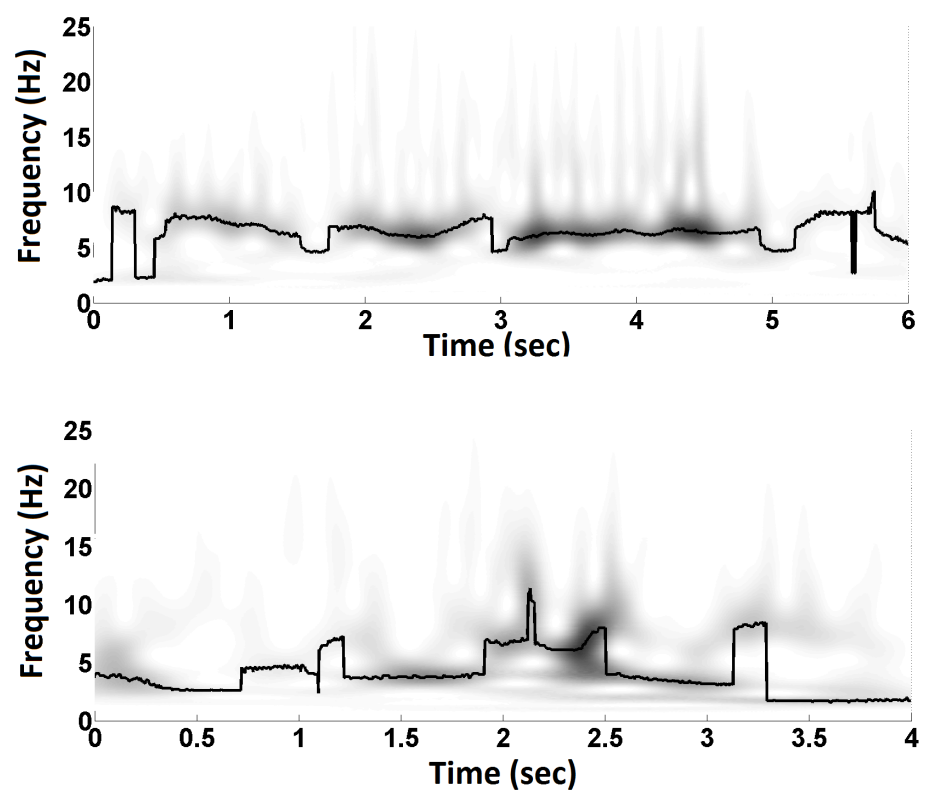

Fig. 2. Ridges (bold lines) of the wavelet spectrograms of epileptic discharge (upper plot) and sleep spindles (lower plot).

Previously the time-frequency segmentation (detection) method of the SS was studied in [8]. In [2] the time-frequency properties of the spike-wave discharges in absence epilepsy with wavelet spectrograms ridges analysis were studied. Start positions of these discharges were selected manually. An approach to automatic detection of SS and ED in PE, based on the analysis of the ridges of the wavelet spectrograms, was developed. Each point of the ridge is the maximum value of 

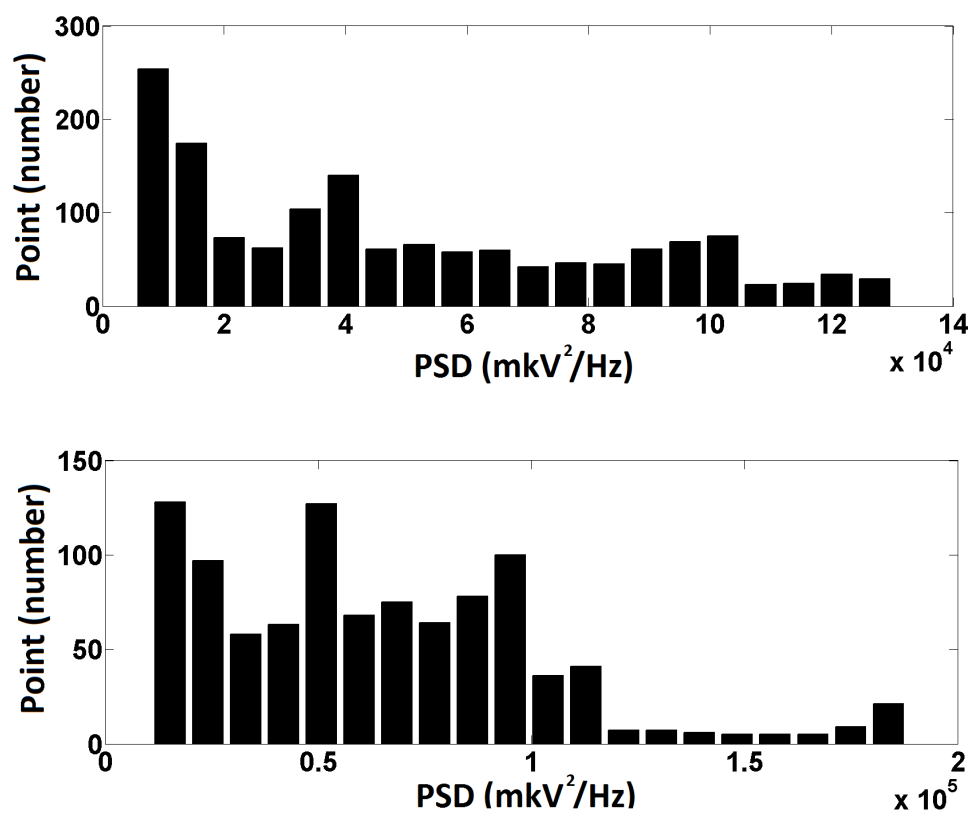

Fig. 3. PSD histograms for the epileptic discharge (upper plot) and sleep spindles (lower plot).

power spectral density in this time point. Examples of wavelet spectrograms and their ridges for ED and SS are shown in figure 2.

SS and ED are characterized by a higher value of the power spectral density in comparison with the background. For the segmentation of SS and ED from background activity, it is advised to analyze histogram of power spectral density at the points of ridge (figure 3 ).

The histograms have a sharp decline in the region of small values of the power spectral density (with $2 * 10^{4}$ in the left graph and $0.4 * 10^{5}$ in the right graph). Therefore, these values of power spectral density can be selected as an adaptive threshold for segmentation of the ED and SS. Areas of the ridge of ED and of SS where the spectral power density is above the PSD threshold value were selected (figure 4).

The following parameters were calculated in selected fragments: frequency range, duration, and the maximum value of power spectral density. These data can be used to classify SS and ED on long EEG-records.

\section{Conclusion}

The following results were obtained: 

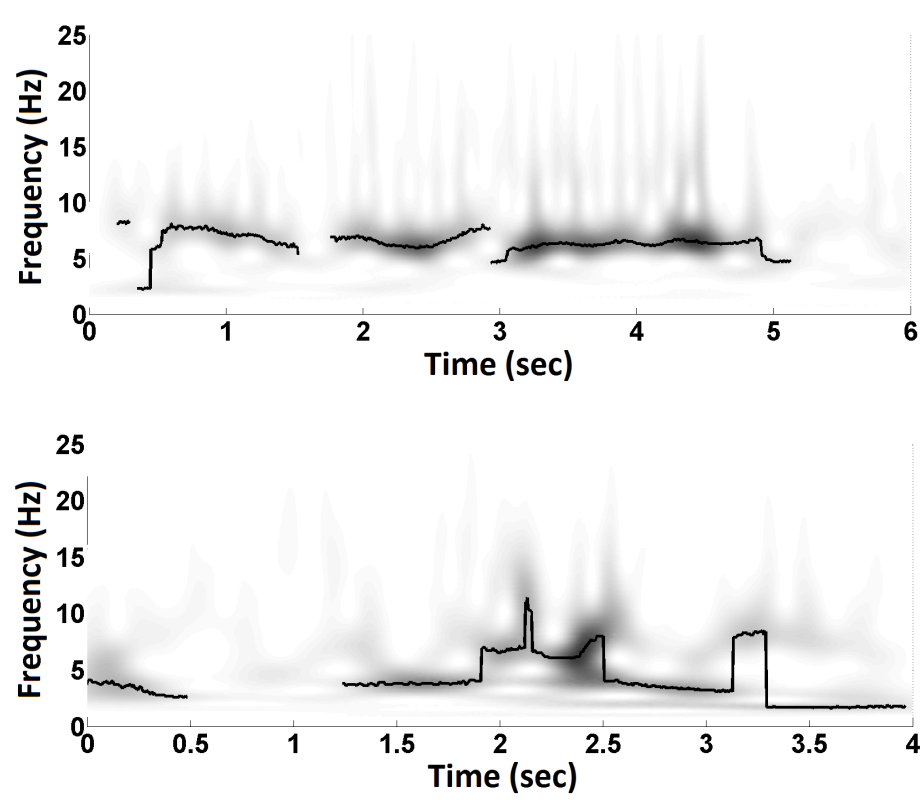

Fig. 4. Wavelet spectrogram with the segmented ridges of epileptiform discharges (upper plot) and sleep spindles (lower plot).

- The algorithm for detection of ED and SS on the background activity was developed.

- 36 EEG records with SS and 39 EEG records with ED were processed using this algorithm.

- Ridge parameters of the processed EEG records were calculated.

Results of this research will help to detect and to separate ED and SS from background activity on long EEG records.

Acknowledgments. This research was done at the expense of the grant of the Russian Scientific Fund (project 16-11-10258)

\section{References}

1. Annegers, J., Hauser, W.A., Coan, S., Rocca, W.: A population-based study of seizures after traumatic brain injuries. NEJM 338, 20-24 (1998)

2. Bosnyakova, D., Obukhov, Y.: Extraction of dominant feature in biomedical signals. Pattern Recognition and Image Analysis 15(3), 513-515 (2005)

3. D'Ambrosio, R., Fender, J., Fairbanks, J., Simon, E., Born, D., Doyle, D., Miller, J.: Progression from frontal-parietal to mesial-temporal epilepsy after fluid percussion injury in the rat. Brain 128(1), 174-188 (2004) 
4. D'Ambrosio, R., Hakimian, S., Stewart, T., Verley, D., Fender, J., Eastman, C., Sheerin, A., Gupta, P., Diaz-Arrastia, R., Ojemann, J., Miller, J.: Functional definition of seizure provides new insight into post-traumatic epileptogenesis. Brain 132(10), 2805-2821 (2009)

5. Goupilland, P., Grossman, A., Morlet, J.: Cycle-octave and related transforms in seismic signal analysis. Geoexploration 23(1), 85-102 (1984)

6. I.Kharatishvili, Pitkanen, A.: Association of the severity of cortical damage with the occurrence of spontaneous seizures and hyperexcitability in an animal model of posttraumatic epilepsy. Epilepsy Research 90(1-2), 47-59 (2010)

7. Kabadi, S., Hilton, G., Stoica, B., Zapple, D., Faden, A.: Fluid-percussion-induced traumatic brain injury model in rats. Nature Protocols 5(9), 1552-1563 (2010)

8. Parekh, A., Selesnick, I., Rapoport, D., Ayappa, I.: Sleep spindle detection using time-frequency sparsity. In: IEEE Signal Processing in Medicine and Biology Symposium (SPMB), 2014 IEEE. pp. 1-6. IEEE, Philadelphia, PA: IEEE. (Dec 2014)

9. Pitkanen, A., Engel, J.: Past and present definitions of epileptogenesis and its biomarkers. Neurotherapeutics 11(2), 231-241 (2014)

10. Pitkanen, A., Immonen, R., Grohn, O., Kharatishvili, I.: From traumatic brain injury to posttraumatic epilepsy: what animal models tell us about the process and treatment options. Epilepsia 50(2), 21-29 (2009)

11. Pitkanen, A., Kharatishvili, I., Karhunen, H., Lukasiuk, K., Immonen, R., Nairismagi, J., Grohn, O., Nissinen, J.: Epileptogenesis in experimental models. Epilepsia 48(2), 13-20 (2007) 